\title{
Plasma lyso-Gb3: a biomarker for monitoring fabry patients during enzyme replacement therapy
}

\author{
Hitoshi Sakuraba ${ }^{1} \cdot$ Tadayasu Togawa $^{2} \cdot$ Takahiro Tsukimura $^{2} \cdot$ Hiroshi Kato $^{3}$
}

Received: 14 October 2017 / Accepted: 17 December 2017 / Published online: 29 December 2017

(c) The Author(s) 2017. This article is an open access publication

\begin{abstract}
Background Recently, globotriaosylsphingosine (lyso-Gb3) has attracted interest as a biomarker of Fabry disease. However, little is known regarding its utility for the evaluation of the therapeutic efficacy.

Method We measured plasma lyso-Gb3 concentration in Japanese healthy subjects and Fabry patients by means of liquid chromatography-tandem mass spectrometry (LC-MS/MS). We determined the reference interval in Japanese (UMIN000016854), and examined the effect of enzyme replacement therapy (ERT) with recombinant $\alpha$-galactosidase A (GLA) and the influence of antibodies against the enzyme on the plasma lyso-Gb3 level in Fabry patients (UMIN000017152). Results The reference interval was determined to be $0.35-0.71 \mathrm{nmol} / \mathrm{L}$, this being almost the same as the normal range in a non-Japanese population previously reported. The analysis revealed that the plasma lyso-Gb3 level was strikingly increased in classic Fabry males, and to a lesser extent in later-onset Fabry males and Fabry females. The elevation of the plasma lyso$\mathrm{Gb} 3$ level was related to renal involvement in the Fabry females. ERT gave a rapid reduction in the elevated plasma lyso-Gb3 level in the classic Fabry males, and a gradual one or stabilization in most of the later-onset Fabry males and Fabry females. However, formation of antibodies against the recombinant GLA had a negative effect on the reduction of plasma lyso-Gb3. Conclusions Regular observation of plasma lyso-Gb3 and antibodies is useful for monitoring of Fabry patients during ERT.
\end{abstract}

Keywords Fabry disease $\cdot$ Globotriaosylsphingosine $\cdot$ Enzyme replacement therapy $\cdot$ Antibody

\section{Introduction}

Fabry disease (OMIM 301500) is an X-linked genetic disorder characterized by deficient activity of $\alpha$-galactosidase A (GLA, EC 3.2.1.22). The enzymatic defect leads to systemic accumulation of glycolipids, predominantly globotriaosylceramide (Gb3) [1]. Fabry disease is divided into three subtypes based on gender and manifestations of a patient. Typical male patients with the "classic" form of Fabry disease exhibit pain in the peripheral extremities, hypohidrosis, angiokeratomas, and corneal opacities during childhood or

Hitoshi Sakuraba

sakuraba@my-pharm.ac.jp

1 Department of Clinical Genetics, Meiji Pharmaceutical University, 2-522-1 Noshio, Kiyose, Tokyo 204-8588, Japan

2 Department of Functional Bioanalysis, Meiji Pharmaceutical University, 2-522-1 Noshio, Kiyose, Tokyo 204-8588, Japan

3 Specialty Medical Affairs Group, Medical Affairs, Sumitomo Dainippon Pharma Co., Ltd., 17-10, Kyobashi 1-Chome,

Chuo-ku, Tokyo 104-0031, Japan adolescence, and develop renal, cardiac, and cerebrovascular involvement in adulthood, and those with the "later-onset" form of the disease show milder manifestations limited to the heart and/or kidneys in adulthood without the childhood symptoms. The manifestations in "Fabry females" are more heterogeneous than in Fabry males ranging from asymptomatic to severe, depending on random X-chromosomal inactivation [1].

Plasma Gb3 has been mainly measured as a biomarker of Fabry disease [2]. However, systemic analysis revealed that $\mathrm{Gb} 3$ was not an ideal biomarker of this disease, because most of the later-onset Fabry males and Fabry females did not exhibit a high plasma Gb3 concentration [3]. Recently, plasma globotriaosylsphingosine (lyso-Gb3) has attracted interest instead of $\mathrm{Gb} 3$ as a surrogate biomarker of Fabry disease [3], and a lot of papers reported that the plasma lysoGb3 level is increased in Fabry patients, especially in classic Fabry males [4-6]. Furthermore, since enzyme replacement therapy (ERT) involving recombinant human GLAs produced in human fibroblasts (agalsidase alfa) and Chinese hamster ovary cells (agalsidase beta) has been successfully 
introduced [7], the importance of biomarkers is increasing more and more for evaluating therapeutic efficacy and monitoring therapy. Some investigators revealed that the elevated plasma lyso-Gb3 level was reduced following ERT and the improvement of some clinical manifestations was associated with it in Fabry patients, suggesting the availability of plasma lyso-Gb3 as an indicator reflecting the efficacy of ERT $[3,5,8]$. On the other hand, it has been reported that antibodies against recombinant GLAs produced in Fabry patients have a negative impact on the therapeutic effect of the enzymes $[9,10]$. However, there have been few studies on the relationship between antibodies and plasma lyso-Gb3. Recently, Rombach et al. investigated the impact of antiGLA antibodies on lyso-Gb3 and the clinical outcome in Fabry patients who had received ERT, and reported that the presence of the antibodies was associated with a less drastic decrease in plasma lyso-Gb3, which may reflect the worse treatment outcome [10]. They emphasized that this issue urgently needed to be addressed and that the findings needed to be confirmed.

Based on their report, we performed a comprehensive study on plasma lyso-Gb3 in Japanese healthy individuals and Fabry patients who had received ERT with agalsidase alfa. In this study, we first determined the reference interval for plasma lyso-Gb3 in Japanese (validation study) and then examined the effect of ERT and the influence of anti-agalsidase alfa antibodies on the plasma lyso-Gb3 concentration in Fabry patients (retrospective study).

\section{Materials and methods}

\section{Reagents}

Lyso-Gb3 was purchased from Matreya, LLC. (Pleasant Gap, PA). Stable-isotope labeled lyso-Gb3, which has one ${ }^{13} \mathrm{C}$ and three deuteriums, was synthesized at Nard Institute, Ltd. (Kobe, Japan) according to the method described previously [6], and used as an internal standard for measurement of plasma lyso-Gb3 (lyso-Gb3-IS). All other reagents used in this study were of analytical grade.

\section{Study subjects and samples}

For the validation study, plasma samples from 100 healthy Japanese subjects (49 males and 51 females; mean age \pm SD, $34 \pm 12$ years; range of age, 15-68 years) were used as samples. They each received a health check-up including family history, past history, and physical and laboratory examinations, and were considered healthy without any evidence of clinical diseases by the physicians working at the healthcare center. The mean plasma GLA activity \pm standard deviation
(SD) was $2.9 \pm 0.7 \mathrm{nmol} / \mathrm{h} / \mathrm{mL}(n=100)$, and none of them exhibited decrease in the enzyme activity.

For the retrospective study to examine the effect of ERT and the influence of antibodies against agalsidase alfa on plasma lyso-Gb3, blood samples from 34 Fabry males (26 classic; mean \pm standard deviation of age, $24 \pm 14$ years; 8 later-onset; $50 \pm 12$ years) and 27 Fabry females (49 \pm 14 years), who received ERT with agalsidase alfa (Replagal $^{\circledR}$; Shire, Lexington, MA; $0.2 \mathrm{mg} / \mathrm{kg} / 2$ weeks) for 0.5-7 years, were obtained at baseline and in subsequent years after the initiation of ERT, and used as samples for the biochemical analysis. All the patients examined in this study are subjects of the specified drug-use survey for agalsidase alfa in Japan, and their phenotypes were determined based on their gender and clinical manifestations described in the survey report. The survey report describes that gene analysis was performed for 29 of the 61 patients, but not for other 32 ones, because their informed consents could not be obtained. The mean plasma GLA activities \pm SD in the classic Fabry males $(n=26)$, the later-onset Fabry males $(n=8)$, and the Fabry females $(n=27)$ were $0.2 \pm 0.5,0.1 \pm 0.1$, and $0.8 \pm 0.4 \mathrm{nmol} / \mathrm{h} / \mathrm{L}$, respectively.

\section{Measurement of lyso-Gb3 in plasma}

Lyso-Gb3 in plasma was measured by liquid chromatography-tandem mass spectrometry (LC-MS/MS). Extraction of lyso-Gb3 from plasma was performed by the modified method described previously [5]. Lyso-Gb3 was dissolved in $\mathrm{CHCl}_{3} / \mathrm{MeOH} /$ water $(2: 1: 0.1)$ and then diluted in $1 \% \mathrm{H}_{3} \mathrm{PO}_{4} /$ $\mathrm{MeOH}$ to prepare solutions of various concentrations of lyso-Gb3 ranging from 0.08 to $250 \mathrm{nmol} / \mathrm{L}$, as standards. As an internal standard (IS), lyso-Gb3-IS was diluted in $1 \%$ $\mathrm{H}_{3} \mathrm{PO}_{4} / \mathrm{MeOH}$ to $2.5 \mathrm{nmol} / \mathrm{L}$. A $50 \mu \mathrm{L}$ aliquot of each standard solution or plasma sample was added to a $200 \mu \mathrm{L}$ aliquot of the IS solution and a $750 \mu \mathrm{L}$ aliquot of $1 \% \mathrm{H}_{3} \mathrm{PO}_{4} / \mathrm{MeOH}$, followed by mixing and centrifugation to remove insoluble proteins. After centrifugation, $1 \mathrm{~mL}$ of each supernatant was transferred to an OASIS MCX cartridge (Waters Co., Milford, MA; $30 \mathrm{mg}, 30 \mu \mathrm{m}$ ) preconditioned with a $1 \mathrm{~mL}$ aliquot of $\mathrm{MeOH}$ and a $1 \mathrm{~mL}$ aliquot of $2 \% \mathrm{H}_{3} \mathrm{PO}_{4}$. The cartridge was washed with a $1 \mathrm{~mL}$ aliquot of $2 \%$ formic acid and then a $1 \mathrm{~mL}$ aliquot of $0.2 \%$ formic acid $/ \mathrm{MeOH}$. Finally, lyso-Gb3 was eluted into a glass tube with a $1 \mathrm{~mL}$ aliquot of $2 \% \mathrm{NH}_{4} \mathrm{OH} / \mathrm{MeOH}$, followed by drying in an evaporator. Each residue was reconstituted in a $200 \mu \mathrm{L}$ aliquot of water/ $\mathrm{CH}_{3} \mathrm{CN}(60: 40, \mathrm{v} / \mathrm{v})$. After sonication and centrifugation, the reconstituted samples were applied to the LC-MS/MS system.

For the LC, a Nexera LC system (Shimadzu, Kyoto, Japan) and a Shim-pak XR-ODS II column (Shimadzu; $100 \mathrm{~mm} \times 3.0 \mathrm{~mm}$ I.D., $2.2 \mu \mathrm{m})$ were used. The mobile phase consisted of formic acid/water $(2: 1,000)$ and $\mathrm{CH}_{3} \mathrm{CN}$. 
Elution was performed in the gradient mode. The flow rate was $0.4 \mathrm{~mL} / \mathrm{min}$ and the injection volume was $10 \mu \mathrm{L}$. The column was maintained at $40^{\circ} \mathrm{C}$. A Triple Quad 6500 mass Spectrometer (AB Sciex Pte., Ltd., Framingham, MA) equipped with an electrospray ionization interface in the positive-ion mode was used for detection of lyso-Gb3. The Multiple Reaction Monitoring (MRM) conditions were optimized with an automatic MRM optimization function. The following transition was monitored in the MRM mode: $\mathrm{m} / \mathrm{z} 786.8 \rightarrow 282.3$ for lyso-Gb3 and $\mathrm{m} / \mathrm{z} 790.8 \rightarrow 286.2$ for lyso-Gb3-IS.

\section{Detection of anti-agalsidase alfa antibodies in serum}

Detection of antibodies against agalsidase alfa in serum from the Fabry patients was performed by enzyme-linked immunosorbent assaying (ELISA), followed by reaction of peroxidase-conjugated goat anti-human $\mathrm{IgG}$ with $o$-phenylenediamine dihydrochloride. Finally, the absorbance at $540 \mathrm{~nm}$ was measured using a microplate reader (SpectraMax Plus 384; Molecular Devices, Sunnyvale, CA). Then, the absorbance ratio at each time point to the baseline in individuals was calculated, and the mean and SD values in the group comprising the later-onset Fabry males and Fabry females (35 patients; number of the time points, $n=149$ ), who were expected to have residual GLA proteins and to hardly form antibodies against the recombinant enzyme, were determined to be 0.73 and 0.29 , respectively. Based on these data, the cut-off point was determined to be 1.60 (mean $+3 \mathrm{SD}$ ), and an absorbance ratio of $\geq 1.60$ was considered to be antibody-positive $(\mathrm{Ab}+)$, and one of $1.60>$ antibody negative $(\mathrm{Ab}-)$, respectively.

\section{Statistical analysis}

Data of the various phenotypic groups are basically expressed as means \pm SD. The differences among the targeted groups were assessed by means of Student's $t$ test, it being taken that there was a significant difference if $p<0.05$. Analyses for variance, assessment of normality, and evaluation of correlation were performed by means of ANOVA, the Shapiro-Wilk test, and Spearman's rank correlation coefficient, respectively.

\section{Results}

\section{Validation study}

To determine the reference interval of the plasma lysoGb3 concentration in Japanese, a validation study was performed. The results revealed that the mean value for plasma
lyso-Gb3 $\pm \mathrm{SD}$ and the median value (minimum-maximum) in the males $(n=49)$ were $0.54 \pm 0.08 \mathrm{nmol} / \mathrm{L}$ and 0.53 (0.39-0.75) nmol/L, respectively, and those in the females $(n=51)$ were $0.53 \pm 0.10 \mathrm{nmol} / \mathrm{L}$ and $0.53(0.31-0.73)$ $\mathrm{nmol} / \mathrm{L}$, respectively. None exhibited an apparently high value. There was no statistical difference in the plasma lyso-Gb3 level between the male and female groups (Student's $t$ test, $p=0.4636)$. Thus, those for all the Japanese subjects ( $n=100,49$ males and 51 females) were calculated to be $0.53 \pm 0.09 \mathrm{nmol} / \mathrm{L}$ and $0.53(0.31-0.75) \mathrm{nmol} / \mathrm{L}$, respectively. As to the relation of the age of the investigated subjects with the plasma lyso-Gb3 level, there was no difference between the young group ( $n=10$, age $<20$ years) and the adult one ( $n=90$, age $\geq 20$ years) (Student's $t$ test, $p=0.6902$ ). The mean plasma lyso-Gb3 \pm SD value and the median value (minimum-maximum) in the young group were calculated to be $0.54 \pm 0.09 \mathrm{nmol} / \mathrm{L}$ and 0.54 (0.40-0.68) nmol/L, respectively, and those in the adult group to be $0.53 \pm 0.09 \mathrm{nmol} / \mathrm{L}$ and $0.53(0.31-0.75)$ $\mathrm{nmol} / \mathrm{L}$, respectively. Thus, based on these data, the reference intervals in Japanese males, females, and all the subjects were determined to be $0.38-0.70,0.33-0.73$, and 0.35-0.71 nmol/L, respectively (Assessment of normality: Shapiro-Wilk test, determination of reference interval: mean $\pm 1.96 \mathrm{SD})$.

\section{Retrospective study}

\section{Pre-treatment plasma lyso-Gb3 concentration in fabry patients}

The plasma lyso-Gb3 concentrations of the Fabry patients and the healthy subjects were measured, and the result was shown in Fig. 1. The mean plasma lyso-Gb3 $\pm \mathrm{SD}$ values were $125 \pm 62 \mathrm{nmol} / \mathrm{L}$ for the classic Fabry males $(n=26$;

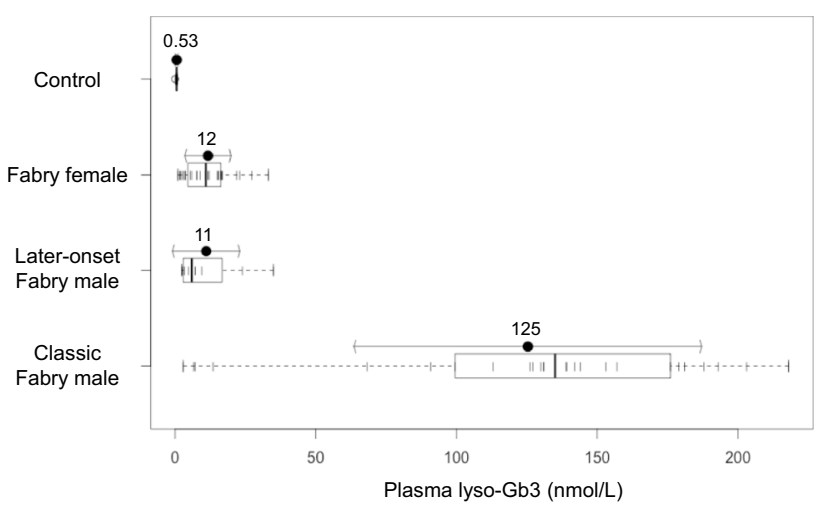

Fig. 1 Comparison of plasma lyso-Gb3 levels between the classic Fabry males, later-onset Fabry males, Fabry females, and the healthy subjects. Data are presented as means \pm SD and box plots with median lines, 25- and 75-percentile boxes 
age $24 \pm 14$ years), $11 \pm 12 \mathrm{nmol} / \mathrm{L}$ for the later-onset Fabry males ( $n=8$; age $50 \pm 12$ years), and $12 \pm 8 \mathrm{nmol} / \mathrm{L}$ for the Fabry females ( $n=27$; age $49 \pm 14$ years). The mean plasma lyso-Gb3 level in the classic Fabry males was significantly higher than that in the healthy controls (Student's $t$ test, $p<0.0001$ ), and those in the later-onset Fabry males and Fabry females were lower than that in the classic Fabry males, but statistically higher than that in the healthy controls (Analysis of variance: ANOVA, Student's $t$ test, $p<0.0001)$.

The relation between the plasma lyso-Gb3 level and the specific life-threatening disease manifestations that the patients exhibited (Renal involvement: proteinuria, renal dysfunction, and renal failure; cardiac involvement: cardiac hypertrophy, arrhythmia, angina pectoris, and cardiomyopathy) was also examined, and the results are summarized in Table 1. The plasma lyso-Gb3 level in the Fabry females who developed renal involvement was statistically higher than in those without this defect (Student's $t$ test, $p=0.0281$ ). There was a tendency that the mean plasma lyso-Gb3 level in the Fabry females who developed cardiac involvement was moderately higher than in those without it, but no statistical difference could be found between the two groups (Student's $t$ test, $p>0.05$ ). Regarding the classic Fabry males, their plasma lyso-Gb3 levels were apparently high regardless of the renal or cardiac manifestations, and such findings were not obtained. For the later-onset Fabry males, statistical evaluation could not be performed, because the number of patients for comparison was too small.

\section{Effect of ERT on the plasma lyso-Gb3 level and impact of antibody formation on plasma lyso-Gb3 reduction following ERT}

Measurement of plasma lyso-Gb3 and detection of antiagalsidase alfa antibodies were performed for the Fabry patients at the baseline, and $0.5,1,2,3,4,5,6$, and 7 years after the initiation of ERT. The time course of the plasma lyso-Gb3 level in each patient with the classic form of Fabry disease is shown in Fig. 2a. In this group, of the 25 patients available for examination, four (Case Nos. 1-4) continuously exhibited an antibody-positive reaction $(\mathrm{Ab}+)$, but two cases (Case Nos. 5 and 6) transiently exhibited a weak response $(\mathrm{Ab}+\rightarrow-)$. The other 19 cases exhibited a negative one ( $\mathrm{Ab}-)$. The plasma lyso-Gb3 level in most of the classic Fabry males, being apparently high at the baseline, significantly decreased following ERT and then remained at a low level, although it did not reach to a value within the reference interval. However, in all the cases that continuously developed anti-agalsidase alfa antibodies, the plasma lyso-Gb3 level apparently increased again and remained at a high level after transient reduction at 0.5 years.

The time course of the plasma lyso-Gb3 level in each male patient with later-onset Fabry disease $(n=8 ; 1 \mathrm{Ab}+$ $\rightarrow-$, Case No.7 and 7Ab-) and each female Fabry patient $(n=27 ; 1 \mathrm{Ab}+\rightarrow-$, Case No. 8 and $26 \mathrm{Ab}-)$ is shown in Fig. 2b, c, respectively. In these cases, plasma lyso-Gb3 moderately decreased or remained at a relatively low level, although it also did not reach to a value within the reference interval.

Based on these data, the effects of ERT on the plasma lyso-Gb3 level for the three phenotypic groups are summarized in Fig. 3a. The mean plasma lyso-Gb3 level in the classic Fabry males, being apparently high at the baseline, quickly and strikingly decreased following ERT. In contrast, those in the later-onset Fabry males and Fabry females, being moderately high at the baseline, gradually and slightly decreased following ERT. Figure $3 \mathrm{~b}$ summarizes the influence of the anti-agalsidase alfa antibodies on the mean plasma lyso-Gb3 level in the classic Fabry male group on ERT. It clearly demonstrated that the presence of the
Table 1 Relation between the plasma lyso-Gb3 level and the specific disease manifestations

\begin{tabular}{|c|c|c|c|c|c|}
\hline & \multirow{2}{*}{$\begin{array}{l}\text { Specific } \\
\text { manifestations }^{\mathrm{a}}\end{array}$} & \multicolumn{2}{|c|}{ Renal involvement } & \multicolumn{2}{|c|}{ Cardiac involvement } \\
\hline & & Age (years) & $\begin{array}{l}\text { Plasma lyso- } \\
\text { Gb3 (nmol/L) }\end{array}$ & Age (years) & $\begin{array}{l}\text { Plasma } \\
\text { lyso-Gb3 } \\
(\mathrm{nmol} / \mathrm{L})\end{array}$ \\
\hline \multicolumn{6}{|l|}{ Fabry males } \\
\hline \multirow[t]{2}{*}{ Classic form } & + & $35 \pm 10(9)$ & $123 \pm 56(9)$ & $34 \pm 11(8)$ & $139 \pm 36(8)$ \\
\hline & - & $18 \pm 12(17)$ & $126 \pm 66(17)$ & $20 \pm 13(18)$ & $119 \pm 70(18)$ \\
\hline \multirow[t]{2}{*}{ Later-onset form } & + & $48 \pm 10(7)$ & $11 \pm 13(7)$ & $49 \pm 14(6)$ & $14 \pm 13(6)$ \\
\hline & - & $68(1)$ & $9(1)$ & $49,58(2)$ & $2,3(2)$ \\
\hline \multirow[t]{2}{*}{ Fabry heterozygous females } & + & $48 \pm 10(10)$ & $16 \pm 10(10)$ & $55 \pm 8(15)$ & $14 \pm 7(15)$ \\
\hline & - & $49 \pm 16(17)$ & $9 \pm 6(17)$ & $41 \pm 17(12)$ & $9 \pm 9(12)$ \\
\hline
\end{tabular}

Values are expressed as means \pm SD (number of examined samples)

${ }^{a}$ Renal involvement (proteinuria, renal dysfunction, and renal failure); cardiac involvement (cardiac hypertrophy, arrhythmia, angina pectoris, and cardiomyopathy) 

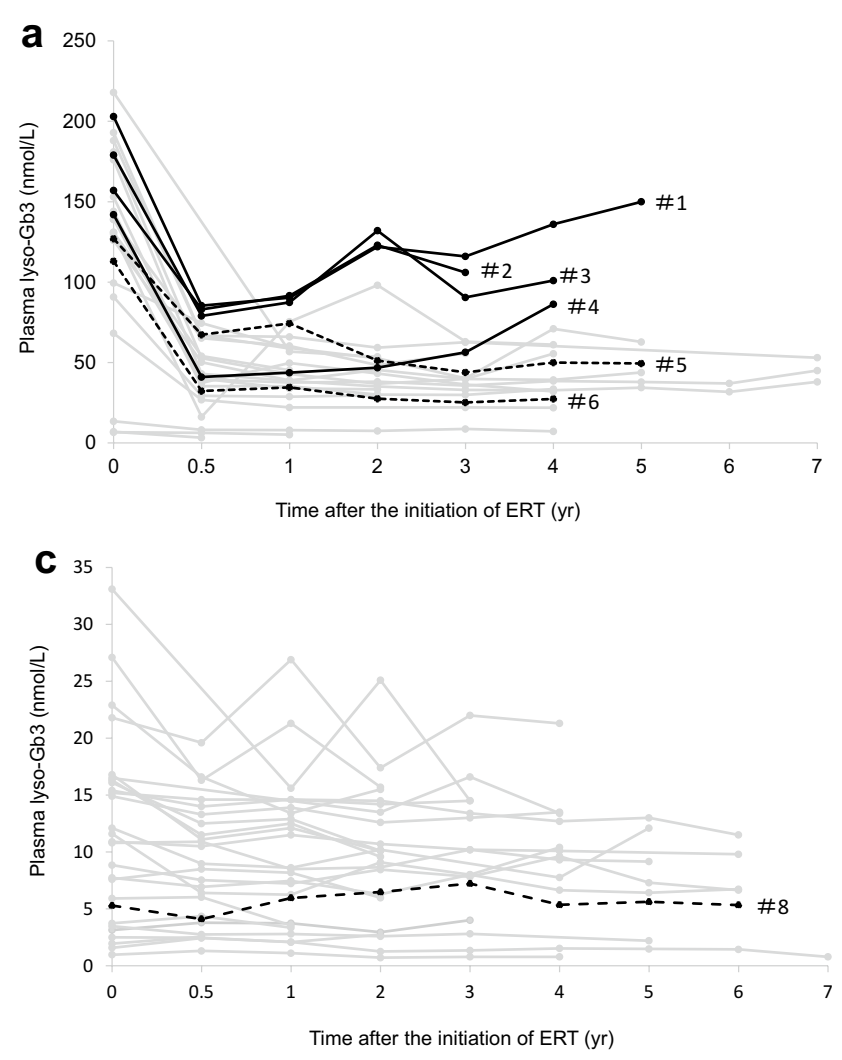

Fig. 2 Time course of the plasma lyso-Gb3 level in each Fabry patient that had received ERT. a Classic Fabry males, b later-onset Fabry males, and c Fabry females. Black lines: Ab+ (Case numbers

antibodies had a negative effect on the reduction of plasma lyso-Gb3 on ERT.

\section{Discussion}

In this study, we tried to determine the reference interval of plasma lyso-Gb3 in Japanese using a sufficient number of proper samples, because it had not been previously determined, although there was a report describing the mean plasma lyso-Gb3 level $\pm \mathrm{SD}$ in apparently healthy subjects $(0.37 \pm 0.11 \mathrm{nmol} / \mathrm{L}, n=40)[6]$. The results revealed that it was $0.35-0.71 \mathrm{nmol} / \mathrm{L}$, this being consistent with the normal range in non-Japanese populations reported by Rombach et al. (0.3-0.5 nmol/L) [10], and Gold et al. $(0.3-0.5 \mathrm{nmol} / \mathrm{L})$ [11].

The pre-treatment plasma lyso-Gb3 level was strikingly increased in the classic Fabry males, and to a lesser extent in the later-onset Fabry males and Fabry females. The elevation of the plasma lyso-Gb3 level was related to the renal involvement in the Fabry females. The reason why there is a statistical difference in the plasma lyso-Gb3 level between the Fabry females who developed renal involvement and those

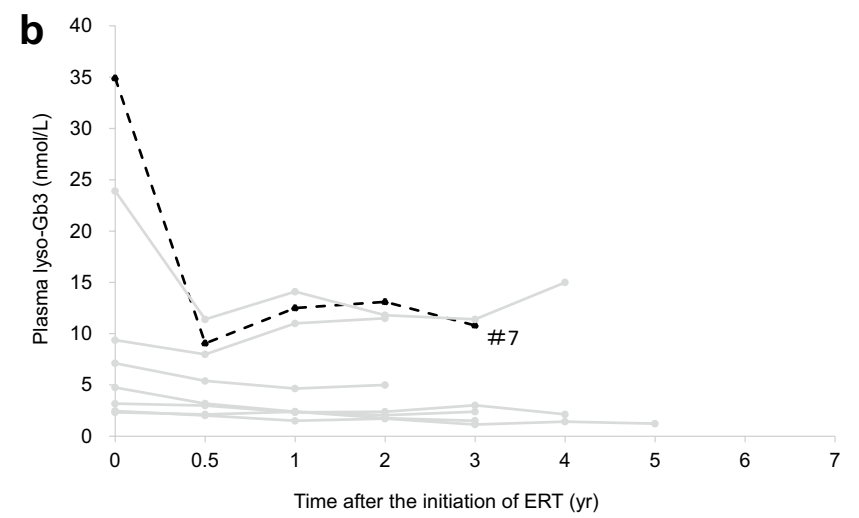

are indicated), black dotted lines: $\mathrm{Ab}+\rightarrow-$ (Case numbers are indicated), and gray lines: Ab-

without the defect is obscure. However, in Fabry females, their clinical manifestations should be influenced not only by the gene mutations which they have but also the distribution of affected cells in organs and tissues based on the random $\mathrm{X}$-chromosomal inactivation, and renal involvement is generally found in the advanced stage of the disease. Considering that lyso-Gb3 promotes release of secondary mediators causing glomerular injury [12], exposure to plasma lysoGb3 may be associated with the progression of the renal disorder. Romback et al. indicated the relation of exposure to plasma lyso-Gb3 with the disease severity and suggested that lyso-Gb3 was a risk factor for the development of left ventricular hypertrophy in female Fabry patients [4].

ERT gave a quick reduction or stabilization of the elevated plasma lyso-Gb3 level, especially in the classic Fabry males. Of the 25 classic Fabry males for whom ELISA data are available, four cases continuously exhibited a positive immune reaction and two cases transiently did so. The formation of antibodies apparently had a negative effect on the reduction of plasma lyso-Gb3, but subjects who gained immune-tolerance escaped from that. In addition, a later-onset Fabry male and a Fabry female who exhibited a transient immune reaction showed a gradual decrease and 
Fig. 3 Change of the mean plasma lyso-Gb3 level in Fabry patients after the initiation of ERT. a Classic Fabry males (black line), later-onset Fabry males (black dotted line), and Fabry females (gray line). b Classic Fabry males exhibiting $\mathrm{Ab}+$ (black line), $\mathrm{Ab}+$ $\rightarrow-$ (black dotted line), and $\mathrm{Ab}-$ (gray line). The data are basically shown as means $\pm \mathrm{SD}$. $N$ number of patients a

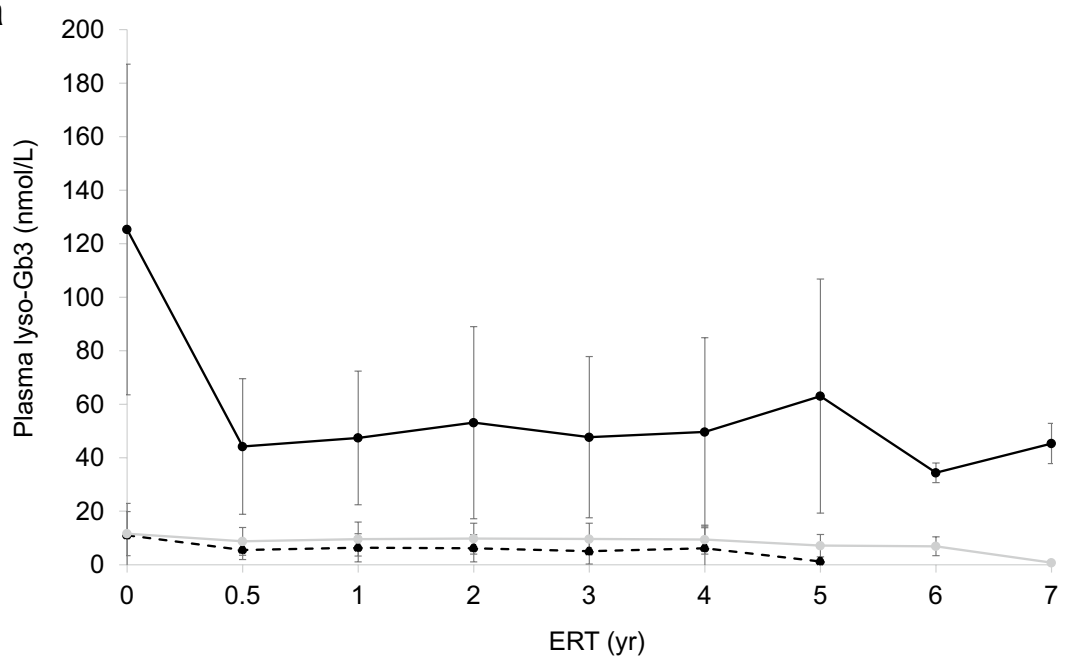

\begin{tabular}{|c|c|c|c|c|c|c|c|c|c|c|}
\hline \multirow{3}{*}{$\mathrm{N}$} & Classic & 26 & 25 & 25 & 21 & 19 & 16 & 6 & 2 & 3 \\
\hline & Later-onset & 8 & 8 & 8 & 8 & 6 & 3 & 1 & 0 & 0 \\
\hline & Female & 27 & 25 & 25 & 24 & 16 & 13 & 8 & 6 & 1 \\
\hline
\end{tabular}

b 250

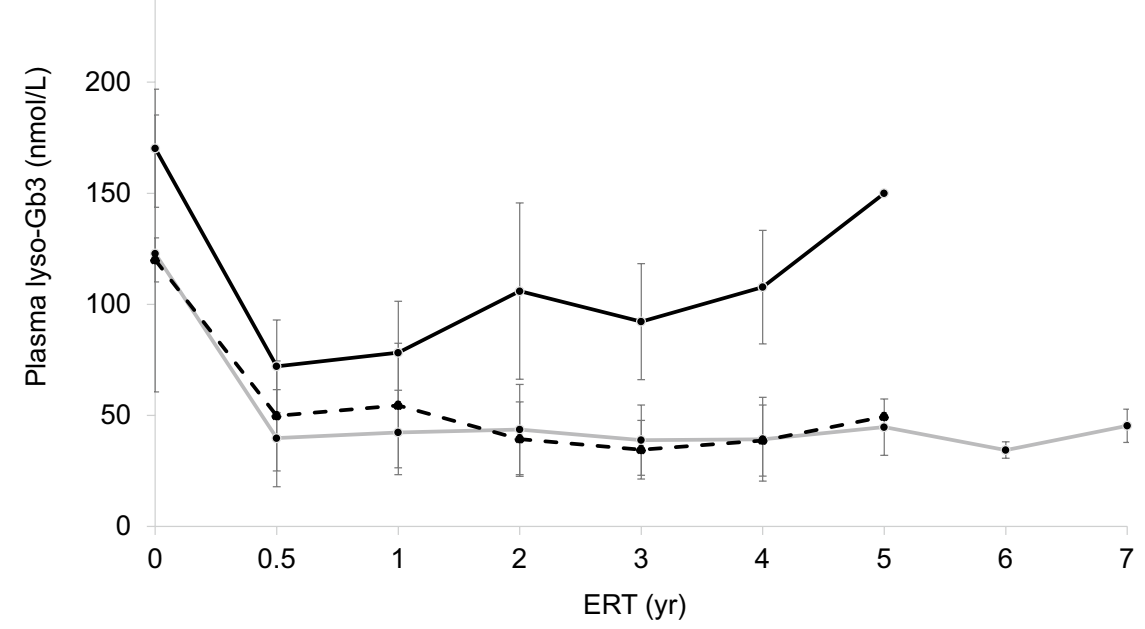

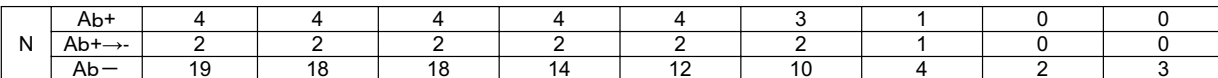

stabilization of the plasma lyso-Gb3 level, respectively, following ERT.

In the initial clinical trials, it was found that antibodies against agalsidase alfa were detected in $21 \%$ of the Fabry males [7], and subsequent studies have revealed that the rate of formation of antibodies against it in Fabry males is $20-56 \%$ [13]. In this study, 21\% (7/33) of the Fabry males exhibited an immune reaction against agalsidase alfa. Differences in the race of the patients examined and/or those in methodology (cut-off point, definition of seropositivity, dilution of samples, timing of assaying, etc.) may influence the results. Unfortunately, genotypes of the antibodypositive cases have not been elucidated yet except for Case
Nos. 4 (p.R227X), 6 (p.W245X), and 7 (p.R301Q), because informed consent for gene analysis could not be obtained from the corresponding patients in the specified drug-use survey previously performed. As the genotype is very important for elucidation of the mechanism of antibody formation [14], we need to determine their genotypes.

In this study, we demonstrated the importance of regular observation of plasma lyso-Gb3 and antibodies against recombinant enzymes during ERT.

Acknowledgements We thank many doctors involved in this study for their provision of the patients' information. This study was supported by Sumitomo Dainippon Pharma Co. Ltd. and the Japan Society for 
the Promotion of Science (JSPS ID: 15K09606, HS), and these were used solely for research activity.

\section{Compliance with ethical standards}

Conflict of interest This study was supported by Sumitomo Dainippon Pharma Co., Ltd. (SDP) and the Japan Society for the Promotion of Science (ID: 15K09606, HS). These were used solely for research activity. Although $\mathrm{H}$. Kato is an employee of SDP, he has no competing interest. We declare that none of the authors have any competing interest.

Human and animal rights This study was approved by the ethical committees of Meiji Pharmaceutical University (IDs 2616 and 2617) and Sumitomo Dainippon Pharma Co., Ltd. (IDs 2015-02 and 2015-03), and was performed according to the ethical guidelines of the 1975 Declaration of Helsinki. The institutional review board or independent ethics committee at each hospital also approved the study.

Registration of clinical trials This study has been registered in UMIN (IDs 000016854 and 000017152).

Informed consent All participants and/or their parents provided written informed consent or assent.

Open Access This article is distributed under the terms of the Creative Commons Attribution 4.0 International License (http://creativecommons.org/licenses/by/4.0/), which permits unrestricted use, distribution, and reproduction in any medium, provided you give appropriate credit to the original author(s) and the source, provide a link to the Creative Commons license, and indicate if changes were made.

\section{References}

1. Germain DP. Fabry disease. Orphanet J Rare Dis. 2010;5:30.

2. Cox TM. Biomarkers in lysosomal storage disease. In: Mehta A, Beck M, Sunder Plassman G, editors. Fabry disease: perspective from 5 years of FOS. Oxford: Oxford PharmaGenesis. 2006;pp. 75-91.

3. Aerts JM, Groener JE, Kuiper S, Donker-Koopman WE, Strijland A, Ottenhoff R, et al. Elevated globotriaosylsphingosine is a hallmark of Fabry disease. Proc Natl Acad Sci USA. 2008;105:2812-7.
4. Rombach SM, Dekker N, Bouwman MG, Linthorst GE, Zwinderman AH, Wijburg FA, et al. Plasma globotriaosylsphingosine: diagnostic value and relation to clinical manifestations of Fabry disease. Biochim Biophys Acta. 2010;1802:741-8.

5. Togawa T, Kodama T, Suzuki T, Sugawara K, Tsukimura T, Ohashi T, et al. Plasma globotriaosylsphingosine as a biomarker of Fabry disease. Mol Genet Metab. 2010;100:257 - 61.

6. Sueoka H, Ichihara J, Tsukimura T, Togawa T, Sakuraba H. Nano-LC-MS/MS for quantification of lyso-Gb3 and its analogues reveals a useful biomarker for Fabry disease. PLoS One. 2015;10:e0127048.

7. Schiffmann R, Kopp JB, Austin HA 3rd, Sabnis S, Moore DF, Weibei T, et al. Enzyme replacement therapy in Fabry disease: a randamized controlled trial. JAMA. 2001;285:2743-9.

8. van Breemen MJ, Rombach SM, Dekker N, Poorthuis BJ, Linthorst GE, Zwinderman AH, et al. Reduction of elevated plasma globotriaosylsphingosine in patients with classic Fabry disease following enzyme replacement therapy. Biochim Biophys Acta. 2011;1812:70 - 6.

9. Lenders M, Stypmann J, Duning T, Schmitz B, Brand SM, Brand E. Serum-mediated inhibition of enzyme replacement therapy in Fabry disease. J Am Soc Nephrol. 2016;27:256 - 64.

10. Rombach SM, Aerts JM, Poorthuis BJ, Groener JE, Donker-Koopman W, Hendriks E, et al. Long-term effect of antibodies against infused alpha-galactosidase A in Fabry disease on plasma and urinary (lyso)Gb3 reduction and treatment outcome. PLoS One. 2012;7:e47805.

11. Gold H, Mirzaian M, Dekker N, Ferraz MJ, Lugtenburg J, Codée JDC, et al. Quantification of globotriaosylsphingosine in plasma and urine of Fabry patients by stable isotope ultraperformance liquid chromatography-tandem mass spectrometry. Clin Chem. 2013;59:547 - 56 .

12. Sanchez-Niño MD, Sanz AB, Carrasco S, Saleem MA, Mathieson $\mathrm{PW}$, Valdivielso JM, et al. Globotriaosylsphingosine actions on human glomerular podocytes: implications for Fabry nephropathy. Nephrol Dial Transplant. 2011;26:1797 - 802.

13. Deegan PB. Fabry disease, enzyme replacement therapy and the significance of antibody responses. J Inherit Metab Dis. 2012;35:227 - 43 .

14. Nakano S, Tsukimura T, Togawa T, Ohashi T, Kobayashi M, Takayama K, et al. Rapid immunochromatographic detection of serum anti- $\alpha$-galactosidase A antibodies in Fabry patients after enzyme replacement therapy. PLoS One. 2015;10:e0128351. 